\title{
Mathematical Properties of Non Static and Non Empty Cosmological Models
}

\author{
R.K. Mishra (Corresponding author) \\ Department of Mathematics, S.L.I.E.T. Longowal \\ Sangrur, Punjab, India
}

Tel: 91-941-711-7990Ｅ-mail: ravkmishra@yahoo.co.in

Amritbir Singh

Department of Mathematics, B.B.S.B. Engineering College

Fatehgarh Sahib, Punjab, India

Tel: 91-991-424-1230Ｅ-mail: singh_amritbir@yahoo.co.in

A.K. Pandey

Department of Mathematics, S.L.I.E.T. Longowal

Sangrur, Punjab, India

Tel: 91-1672-305-264Ｅ-mail: arunesh11pandey@yahoo.co.in

\begin{abstract}
In the present paper we have discussed the Mathematical Properties of non static and non empty cosmological models with variable Cosmological constant $(\Lambda) \&$ variable Gravitational constant $(\mathrm{G})$ under some suitable assumptions. It may be concluded that the Cosmological constant $(\Lambda)$ may be replaced by a non-linear scalar field. It has been also focused that there is close relation between model with scalar field \& model with perfect fluids whose equation of state is more or less exotic.
\end{abstract}

Keywords: Cosmological Model, Non-static universe, Scalar field

\section{Introduction}

At the time of Einstein, the Cosmological Constant $\Lambda$ was having no physical meaning but recent Cosmological observations indicate that the expansion of the universe is accelerating and this has led to a great deal of theoretical activities. Non static cosmological expansion raises a variety of interesting mathematical questions different with kinds of non-empty universes. Here in the present paper we have tried to investigate the mathematical properties which have been discussed up to now and many other challenges which have been left and require attention to solve by peer researchers. We have also tried to discuss the kind of non-empty universes with according to contemporary classification of cosmological models as proposed by (Berman 1991, p. 465-469, Rahman 1990, p. 655-663) under suitable assumptions given by Mishra. (Pande 2000, p. 161-175), (Mishra 2004 p. 202-209).

Non-static expansion plays a role in cosmology in two different regimes. The first in the very early universe while the second in the period between the decoupling of the microwave background radiation and the present scenario. Non-static expansion in the early universe is associated with the name inflation (Guth 1981, p. 347-356), (Mishra 2009, p. 101-104). One of the interested aspects of the inflation is that it claimed to solve certain queries in cosmology but we feel that this could not satisfy the queries related with:

- Homogeneous \& Isotropic nature of universe

- Flatness Problem

- Horizon Problem

Recently, a very strong observational evidence came into picture that the velocity of recession of distinct galaxies is accelerating. This is associated with Dark Energy introduced by (Caldwell 1998, p.1582-1585). After decoupling we have number of different evidences for cosmic acceleration as:

1. Supernovae of Type Ia

2. Microwave background fluctuation 
3. Gravitational lensing

4. Galaxy clustering

In a separate communication data of the above evidences has been discussed by the authors.

\section{Kinds of Non-empty universes}

Here we will try to discuss the list of kinds of non-empty universes according to the Friedmann et al. (Isham 1973, p. 244), (Sivaram 1974 p. 249).

\subsection{Einstein's Models or static models}

$$
\frac{\ddot{R}}{R}=-\frac{k c^{2}}{2}\left(\frac{\rho}{3}+\frac{p}{c^{2}}\right)+\frac{\Lambda c^{2}}{3}
$$

or

$$
\frac{3 \dot{R}^{2}}{R^{2}}+\frac{3 k c^{2}}{R^{2}}=k \rho c^{2}+\Lambda c^{2}
$$

In this case $\mathrm{R}$ remains unchanged and the above equation (1) gives

$$
\begin{gathered}
\Lambda=\frac{k}{2}\left(\rho+\frac{3 p}{c^{2}}\right) \\
\frac{3 k}{R^{2}}=k \rho+\Lambda
\end{gathered}
$$

Consequence of this is that $\rho \& \mathrm{p}$ remain unchanged as well and that $\Lambda>0, k=+1$. This model is unstable because due to change in one or two of the quantities $\mathrm{R}, \rho$, and $\mathrm{p}$, the model compresses themselves up to the special state of the infinite density. Eddington was the first, who pointed out the instability of the Einstein model (Eddington 1930, p. 668), after that many peer researchers has also pointed out the same.

\subsection{Models of the first kind}

Here we present asymptotic, monotone and oscillating models.

\subsubsection{Asymptotic models}

Asymptotic models of the first kind change their volumes monotonically during their expansion or contraction between the special state of infinite density, when $t=t_{0} \&$ a static state, when $t \rightarrow-\infty$ or $+\infty$.

This is possible under $\Lambda>0 \& k=+1$.

\subsubsection{Monotone models}

Monotone models of first kind change their volume monotonically during their expansion between the special state of the infinite density, when $t=t_{0} \&$ infinite cleanliness, when $t \rightarrow-\infty$ or $t \rightarrow+\infty$.

This is under $\Lambda>0, k=0 \& k=-1$.

2.2.3 Oscillating models

Oscillating models of the first kind expands their volume from the special state, when $t=t_{1}$ continue expansion up to $t=t_{0}$.

This is possible with $\Lambda \geq 0$ under $k=+1$ or with $\Lambda<0$ under any numerical value of $\mathrm{k}$.

\subsection{Models of the second kind}

\subsubsection{Asymptotic models of the second kind}

Asymptotic models of the second kind change their volume during their monotone expansion or compression between a static state, corresponding to the Einstein model, when

$t \rightarrow-\infty$ or $t \rightarrow+\infty$ under $\Lambda>0 \& k=+1$.

\subsubsection{Monotone models of second kind}

Monotone models of second kind compress their volumes from the state of infinite cleanliness when $t \rightarrow-\infty$, up to a state of their maximal volume when $t=t_{0}$ under $\Lambda>0 \& k=1$.

So we may conclude that in case of $p>0$ or $p=0$, the possible kinds of the models may be shown as in Table 1 (placed on the last page of the paper). 


\section{Nature \& Analysis of the Models}

\section{When $\Lambda>0$}

a) In his paper, Starobinsky has discussed about expansions for the late-time behavior of spacetime (Starobinsky 1983, p. 66-67). He studied the case where the matter is described by a perfect fluid with linear equation of state $p=(\gamma-1) \rho$, where $1 \leq \gamma \leq 2$. He has also discussed the vacuum case which, as it turns out, gives the leading order terms in the expansion of the geometry for the case with perfect fluid as well. We can write the De-Sitter solution as :

$$
e^{2 H t}\left(d x^{2}+d y^{2}+d z^{2}\right)-d t^{2}
$$

where

$$
H=\sqrt{\frac{\Lambda}{3}}
$$

The expansion discussed by Starobinsky in (Starobinsky 1983, p. 66-67) is expressed in terms of Gauss coordinates.

b) In the reference of power series of the vacuum Einstein equations with $\Lambda>0$, this has been discussed by Rendall (Rendall 2004) with the result

$$
g_{i j}=e^{2 H t}\left[g^{0}{ }_{i j}+\sum_{k=1}^{\infty} \sum_{l=0}^{L_{k}}\left(g_{i j}\right)_{k-2, l} l^{l} e^{-k H t}\right]
$$

where

$$
H=\sqrt{\frac{2 \Lambda}{n(n-1)}}
$$

In this communication the generality of the solutions has been discussed and verified by using counting function. Further it is mentioned that the theorem discussed by Wald shows that for space-times of Bianchi types I - VIII, $\Lambda>0 \& t \rightarrow \infty$ (Wald 1983, p. 2118-2120). This means that the asymptotic of these spacetimes have some of the properties which follow from the asymptotic expansions. There may be also a class of highly symmetric inhomogeneous spacetime with $\Lambda>0$, for which global and asymptotic properties has been established for the large initial data. In all the above results with $\Lambda>0$, where $\Lambda$ expand forever. This indicates the isotropization at late time. At first instance it seems that the spacetime dose not become homogeneous at late times, since the coefficient $g^{0}{ }_{i j}$ of the leading term in the expansion is not homogeneous, so we can say that a positive cosmological constant leads to the solutions of the Einstein equations which look like De-Sitter space on a long time interval.

\section{Connection between fluids \& fields}

If we consider the dependence of the pressure in a homogeneous and isotropic model of the universe as:

$$
p=\beta \rho c^{2}
$$

Where $\beta$ is the radiation, $\rho$ is the density \& $\mathrm{c}$ is the velocity of light. For $\beta=0 ; p=0$, the model is pressure less, also for $\beta=\frac{1}{3} ; p=\frac{\rho c^{2}}{3}$, the model is filled with radiation.

In usual practice, a model is constructed in two ways either considering $\beta=0$ or $\beta=\frac{1}{3}$. The models constructed with $\beta=0$ are used to study the universe at present epoch and the models constructed with $\beta=\frac{1}{3}$ are used to study the universe when it was radiation dominated. In this way we do not have a theory how the universe reaches to matter dominated era from the radiation dominated era. In matter dominated era a model frequently used to produce accelerated expansion is a perfect fluid which isolates the strong energy condition.

The equation of state is

$$
p=(\gamma-1) \rho
$$

but

$$
\gamma<1 \Rightarrow \frac{d p}{d \rho}<0
$$

So it means speed of sound becomes imaginary. Now we can consider that a potential state of the corresponding nonlinear scalar field can reproduce the fluid solution. Since we know the energy momentum tensor of the scalar field as:

$$
T_{i j}=\nabla_{i} \theta \nabla_{j} \theta-\left[\frac{1}{2} \nabla^{r} \theta \nabla_{r} \theta+P(\theta)\right] g_{i j}
$$


Where $\mathrm{P}$ is a smooth non-negative function (the potential).

$$
\dot{\theta}^{2}=\frac{2 \gamma}{2-\gamma} P
$$

since, $P \neq \rho \Rightarrow \dot{\theta} \neq 0$, so it may be cancelled.

$$
\ddot{\theta}=\frac{\gamma}{2-\gamma} P^{\prime}(\theta)
$$

Solving the above equations in different cases, we have the range of the values of $\mathrm{k}$, for power-law inflation it may be related with $\gamma$ as

$$
k=\sqrt{3 \gamma}
$$

The quantities p \& $\rho$ defined here from the scalar field satisfy the Euler expansions since the energy momentum tensor of the scalar field is divergence-free. Hence they agree with the fluid density and pressure everywhere. But this procedure does not extend to models which are homogeneous but not isotropic.

\section{Metric and Properties of Matter}

The space is a constant curvature space having homologous expansions and compressions with

$$
C=\frac{3 k}{R^{2}}, \quad k=0,-1,+1 \quad \& \quad R=R(t)
$$

Euclidean spatial coordinates has the metric

$$
d s^{2}=c^{2} d t^{2}-R^{2} \frac{d x^{2}+d y^{2}+d z^{2}}{\left[1+\frac{k}{4}\left(x^{2}+y^{2}+z^{2}\right)\right]^{2}}
$$

Case i) $k=+1$, had first been considered by Einstein for his static model, and by Friedman for the non-static models.

Case ii) $k=0$, had first been considered by de Sitter for the empty static model, by Lamattre for the non-empty non-static model and by Robertson for the non empty models.

Case iii) $k=-1$, had first been considered by Friedmann since from geometry, it is clear that,a space of $k=+1$ is locally spherical, a space of $k=0$ is locally euclidean and a space of $k=-1$ is locally hyperbolic.

Assuming spherical symmetry in respect of any point and applying it to the properties of coherence, we got that the space of $k=+1$ is elliptic or spherical and euclidean or hyperbolic respectively.

Let us consider $g_{\alpha \beta}$ and putting it into the Einstein equations of gravitations we have:

$$
G^{i j}-\frac{1}{2} g^{i j} G=-k T^{i j}-\Lambda g^{i j}
$$

We obtain that

$$
\rho=\rho(t) \& p=p(t)
$$

exists where $T^{i j}$ can be expressed by the formulas

$$
\begin{gathered}
T^{00}=\frac{1}{g_{00}}\left(\rho+\frac{p}{c^{2}}\right)-\frac{p}{c^{2}} g^{00} \\
T^{0 j}=-\frac{p}{c^{2}} g^{0 j} \\
T^{i k}=-\frac{p}{c^{2}} g^{i k}
\end{gathered}
$$

in other words

$$
T^{i j}=\left(\rho+\frac{p}{c^{2}}\right) \frac{d x^{i}}{d s} \frac{d x^{j}}{d s}-\frac{p}{c^{2}} g^{i j}
$$

Consider four- dimensional velocity, which characterizes the mean motion of a matter in the neighborhood of every point. The pioneer work was done in relativistic cosmology by Einstein and Friedmann, they did not take the pressure into account, Friedmann was the first who considered the static models, so the case of $\rho>0, p=0$ is known as Friedmanns case in inhomogeneous universe. The first, Who introduce $p>0$ was Lemaitre. In an open universe, the sum of the angles 
of a triangle is lower than $180^{\circ}$. In a closed universe (like the surface of the Earth), this sum is always greater than $180^{\circ}$ as shown in Figure-I (placed on the last page of the paper).

\section{Concluding Remarks}

This paper provides the mathematical analysis of cosmological models with non-static (accelerated) expansion. The exposition with a review of results in the case of positive cosmological constant has been discussed in brief and it is highlighted that the cosmological constant $\Lambda$ may be replaced by a non-linear scalar field. It has been also focused that there are close relations between models with scalar field \& models with perfect fluids whose equation of state is more or less exotic. In this paper the universe expansion has been discussed as proposed by Starobinsky and it may be concluded that the generality of the solutions may be verified by using counting function. This result may also be extended for fluids with linear equation of state in $3+1$ dimension. Therefore an efficient check with the above discussed assumptions in suitable framework, which might be the effective behavior common to a large class of high energy theories may be investigated in the separate communication in the near future.In an open universe, the sum of the angles of a triangle is lower than $180^{\circ}$.In a closed universe (like the surface of the Earth), this sum is always greater than $180^{\circ}$.

\section{Acknowledgment}

The authors would like to thank the external reviewers for the suggestions and it was helpful during revision of the manuscript.

\section{References}

Berman. (1991). Cosmological models with variable gravitational and cosmological "constants". General Relativity and Gravitation, 23, 4, 465-469.

Caldwell R.R., Dave R., and Steinhardt P.J. (1998). Cosmological imprint of an energy component with general equation of state. Phys. Rev. Lett., 80, 1582-1585.

Eddington A.S. (1930). On the instability of Einstein's spherical world. Mon. Not. Roy. Astron. Soc., $90,668$.

Guth A. (1981). Inflationary universe: A possible solution to the horizon and flatness problems. Phys. Rev., D23, $347-356$.

Isham C.J., Salam A. \& Strathdee J. (1973). The influence of f Gravity on Gravitational collapse. Nature Phys.Sci., 82, 244.

Sivaram C., Sinha K.P. \& Lord E.A. (1974). The Role of f gravity in cosmological models.Nature, 249, 640-641.

Mishra R.K. \& Singh A. (2009). Variable Cosmology with "big-rip". European Astronomical Society Publication SereisEDP Sciences, 36, 101-104.

Mishra Ravi Kant \& Chandra R. (Dec. 2004). Cosmological models with $\rho G \propto H^{2}$ and $\Lambda \propto H^{2}$, The Mathematical Education, Volume XXXVIII, No. 4, 202-209.

Pande, Chandra \& Mishra. (Feb. 2000). Cosmological Models with variable Cosmological constant and Gravitational constant. Ind. J. Pure and Appl. Math., 31, (2).

Rahman A. (1990). A critical density model with varying gravitational and cosmological "constants". General Relativity and Gravitation, 22, 6, 655-663.

Rendall A.D. (2004). Mathematical properties of cosmological models with accelerated expansion. Electronic archive www.arxiv.org/gr-qc/0408072.

Starobinsky A.A. (1983). Isotropization of Arbitrary Cosmological Expansion Given an Effective Cosmological constant. JETP Lett., 37, 66-67.

Wald R.M. (1983). Asymptotic behaviour of homogeneous cosmological models with cosmological constant. Phys. Rev., D28, 2118-2120. 
Table 1. Different types of models

\begin{tabular}{|c|c|c|c|}
\hline $\begin{array}{l}k \rightarrow \\
\downarrow\end{array}$ & $\mathbf{k}=\mathbf{- 1}$ & $\mathbf{k}=\mathbf{0}$ & $\mathbf{k}=+\mathbf{1}$ \\
\hline$\Lambda>0$ & $\begin{array}{c}\text { Monotone Models } \\
\text { of first kind }\end{array}$ & $\begin{array}{c}\text { Monotone Models } \\
\text { of first kind }\end{array}$ & $\begin{array}{c}\text { (i)Asymp. Models of I and II kind } \\
\text { (ii)Monotone Models of I and II kind } \\
\text { (iii)Oscillating models of I kind }\end{array}$ \\
\hline$\Lambda=0$ & $\begin{array}{c}\text { Oscillating models } \\
\text { of I kind }\end{array}$ & $\begin{array}{c}\text { Monotone models } \\
\text { of first kind }\end{array}$ & $\begin{array}{c}\text { Monotone models } \\
\text { of first kind }\end{array}$ \\
\hline$\Lambda<0$ & $\begin{array}{c}\text { Oscillating models } \\
\text { of first kind }\end{array}$ & $\begin{array}{c}\text { Oscillating models } \\
\text { of first kind }\end{array}$ & $\begin{array}{c}\text { Oscillating models } \\
\text { of first kind }\end{array}$ \\
\hline
\end{tabular}

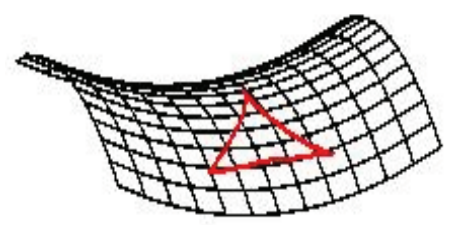

Open universe :

looks like a

horse saddle
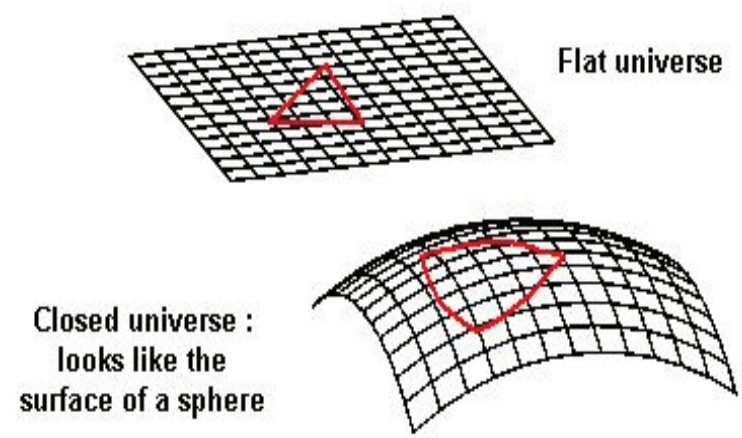

Figure 1. This is an example about figures 